\title{
Quenching for Porous Medium Equations
}

\author{
Burhan Selcuk
}

\begin{abstract}
This paper studies the following two porous medium equations with singular boundary conditions. First, we obtain that finite time quenching on the boundary, as well as $k_{t}$ blows up at the same finite time and lower bound estimates of the quenching time of the equation

$$
k_{t}=\left(k^{n}\right)_{x x}+(1-k)^{-\alpha},(x, t) \in(0, L) \times(0, T)
$$

with $\left(k^{n}\right)_{x}(0, t)=0,\left(k^{n}\right)_{x}(L, t)=(1-k(L, t))^{-\beta}, t \in(0, T)$ and initial function $k(x, 0)=k_{0}(x), x \in[0, L]$ where $n>1, \alpha$ and $\beta$ are positive constants. Second, we obtain that finite time quenching on the boundary, as well as $k_{t}$ blows up at the same finite time and a local existence result by the help of steady state of the equation

$$
k_{t}=\left(k^{n}\right)_{x x},(x, t) \in(0, L) \times(0, T)
$$

with $\left(k^{n}\right)_{x}(0, t)=(1-k(0, t))^{-\alpha},\left(k^{n}\right)_{x}(L, t)=(1-k(L, t))^{-\beta}, t \in(0, T)$ and initial function $k(x, 0)=k_{0}(x), x \in[0, L]$ where $n>1, \alpha$ and $\beta$ are positive constants.
\end{abstract}

\section{Introduction}

The porous medium equations appear in different branches of applied sciences where this simple model appears in a natural way. It has been used to model fluid flow, chemical reactions, heat transfer, diffusion, population dynamics, and so on. Nonlinear diffusion equations involving the porous medium equations have been considered comprehensively (cf. the book by Vazquez [10] and [2], [3], [6], [9], [11]). In literature, blow-up problem is studied rather than quenching problem for porous medium equations. Jiang, et. al. [3] studied to get blow-up properties in the following porous medium equation

$$
\left\{\begin{array}{l}
k_{t}=\left(k^{n}\right)_{x x}+k^{\alpha},(x, t) \in(0,1) \times(0, T), \\
\left(k^{n}\right)_{x}(0, t)=0,\left(k^{n}\right)_{x}(1, t)=k^{\beta}(1, t), t \in(0, T), \\
k(x, 0)=k_{0}(x), x \in[0,1],
\end{array}\right.
$$

2010 Mathematics Subject Classification. 35K05,35K20,35K57,35B50.

Key words and phrases. Maximum principles, Nonlinear diffusion equation, Heat equation, Quenching, Singular boundary condition.

Corresponding author: Burhan Selcuk. 
where $n>1, \alpha, \beta>0$, and $T \in(0, \infty)$. They obtained that finite-time blow up occurred if and only if $\max (\alpha, \beta)>1$, and blow up rate by using certain conditions. Recently, it has been considered to get quenching properties of various reaction-diffusion equations with two singular boundary conditions ([1], [4], [5], [7], [8], [9], [12], [13]). Among them, in [1], Chan and Yuen studied the following problem

$$
\left\{\begin{array}{l}
k_{t}=k_{x x},(x, t) \in(0, a) \times(0, T), \\
k_{x}(0, t)=(1-k(0, t))^{-\alpha}, k_{x}(a, t)=(1-k(a, t))^{-\beta}, t \in(0, T), \\
k(x, 0)=k_{0}(x), 0 \leq k_{0}(x)<1, x \in[0, a],
\end{array}\right.
$$

where $T \leq \infty$ and $\alpha, \beta>0$. They showed that the finite-time quenching occured on the boundary and $k_{t}$ blowed up by using certain assumptions. Further, they achieved to get non-quenching criteria and quenching criteria using steady state. Selcuk and Ozalp [5] studied the following problem:

$$
\left\{\begin{array}{l}
k_{t}=k_{x x}+(1-k)^{-\alpha},(x, t) \in(0,1) \times(0, T), \\
k_{x}(0, t)=0, k_{x}(1, t)=(1-k(1, t))^{-\beta}, t \in(0, T), \\
k(x, 0)=k_{0}(x), x \in[0,1]
\end{array}\right.
$$

where $T \leq \infty$ and $a, \alpha, \beta>0$. They showed that the finite-time quenching occured on the boundary and $k_{t}$ blowed up by using certain assumptions as in [1]. Further, they achieved estimates for the quenching time. Zhi and $\mathrm{Mu}$ [12] studied the following problem with different sources

$$
\begin{aligned}
& k_{t}=k_{x x}+(1-k)^{-\alpha},(x, t) \in(0,1) \times(0, T), \\
& k_{x}(0, t)=k^{-\beta}(0, t), k_{x}(1, t)=0, t \in(0, T), \\
& k(x, 0)=k_{0}(x), x \in[0,1],
\end{aligned}
$$

where $T \leq \infty$ and $\alpha, \beta>0$. They showed that the finite-time quenching occurred on the boundary and achieved the quenching rate estimates is $(T-t)^{1 / 2(\beta+1)}$ if $T$ denotes the quenching time. Zhu [13] studied the following problem with same sources as in [5]:

$$
\begin{aligned}
& k_{t}=\left(\left|k_{x}\right|^{p-2} k_{x}\right)_{x}-k^{-\alpha},(x, t) \in(0,1) \times(0, T), \\
& k_{x}(0, t)=k^{-\beta}(0, t), k_{x}(1, t)=0, t \in(0, T), \\
& k(x, 0)=k_{0}(x), x \in[0,1],
\end{aligned}
$$

where $T \leq \infty$ and $p>1, \alpha, \beta>0$. They showed that the finite-time quenching occurred on the boundary. They also achieved estimates for the quenching rate and the quenching time.

In this paper, we consider the following two porous medium equations with two singular sources to obtain quenching properties extracted from the solutions:

$$
\left\{\begin{array}{l}
k_{t}=\left(k^{n}\right)_{x x}+(1-k)^{-\alpha},(x, t) \in(0, L) \times(0, T), \\
\left(k^{n}\right)_{x}(0, t)=0,\left(k^{n}\right)_{x}(L, t)=(1-k(L, t))^{-\beta}, t \in(0, T), \\
k(x, 0)=k_{0}(x), x \in[0, L],
\end{array}\right.
$$


and

$$
\left\{\begin{array}{l}
k_{t}=\left(k^{n}\right)_{x x},(x, t) \in(0, L) \times(0, T), \\
\left(k^{n}\right)_{x}(0, t)=(1-k(0, t))^{-\alpha},\left(k^{n}\right)_{x}(L, t)=(1-k(L, t))^{-\beta}, t \in(0, T), \\
k(x, 0)=k_{0}(x), x \in[0, L]
\end{array}\right.
$$

where $n>1, \alpha, \beta, L>0$ and $T \in(0, \infty)$. Let $k_{0}(x)$ be a positive initial function satisfying the compatability conditions for (1.4) and (1.5).

Now, we give definition of quenching for the solution of (1.4) and (1.5). Let, $T_{\beta}$ be a finite quenching time and $\gamma$ be a quenching point with $\gamma \in[0, L]$. The solution of (1.4) and (1.5) are called quenching such that

$$
\lim _{t \rightarrow T_{\beta}^{-}} \sup k(\gamma, t) \rightarrow 1 \text {. }
$$

Motivated by (1.1),(1.2) and (1.3), we consider to get the quenching properties of (1.4) and (1.5). In Section 2, we obtain that single quenching point is $x=L$ in finite time and $k_{t}$ blows up at the same time by using the certain assumptions in (1.4). Also, we obtain that lower bound estimates for quenching time by using lower solutions of (1.4). In Section 3, we obtain that single quenching point is $x=L$ in finite time and $k_{t}$ blows up at the same time by using certain assumptions (1.5). Finally, we get a result for local existence using steady state of (1.5).

\section{Quenching for (1.4)}

In this section, we assume that $k_{0}(x)\left(h_{0}(x)\right)$ satisfies the following inequalities

$$
\begin{aligned}
& k_{x x}^{n}(x, 0)+(1-k(x, 0))^{-\alpha} \geq 0\left(h_{x x}(x, 0)+\left(1-h^{1 / n}(x, 0)\right)^{-\alpha} \geq 0\right), \\
& k_{x}(x, 0)>0\left(h_{x}(x, 0)>0\right) .
\end{aligned}
$$

Also, we can easily prove the existence of positive local solution of (1.4) for some $T>0$ using assumption (2.2) as proved in [6].

\subsection{Quenching properties}

Firstly, one can easily show that $k_{0}(x)$ satisfying (2.1), (2.2) and compatibility conditions. Indeed, we assume that the conditions (2.1) and (2.2) are proper.

Remark 1. $k_{0}(x)=\frac{5}{4} x^{\frac{5}{8}}$ satisfies compatibility conditions, (2.1) and (2.2), where $n=2, \beta=$ $0.29827, L=1 / 2$.

Remark 2. If we suppose (2.1) and (2.2) hold and use the maximum principles, then we have $k_{t}>0$ and $k_{x}>0$ in $(0, L] \times(0, T)$, respectively. (See Lemma 2.1 in [11]) 
Theorem 2.1. $k$ quenches in a finite time $T_{\beta}$ if $k_{0}(x)$ satisfies (2.1).

Proof. Suppose that $k_{0}(x)$ satisfies (2.1). Thus, we obtain

$$
\Theta=(1-k(L, 0))^{-\beta}+\int_{0}^{L}(1-k(x, 0))^{-\alpha} d x>0 .
$$

Let's define an auxiliary function; $Z(t)=\int_{0}^{L}(1-k(x, t)) d x, 0<t<T$. In that case, we obtain

$$
Z^{\prime}(t)=-(1-k(L, t))^{-\beta}-\int_{0}^{L}(1-k(x, t))^{-\alpha} d x \leq-\Theta,
$$

from Remark 2. Hence, $Z(t) \leq Z(0)-\Theta t$. Namely, $Z\left(T_{\beta}\right)=0$ for some $T_{\beta}=Z(0) / \Theta$, which means quenching occurs in finite time $T_{\beta}$ for $0<T \leq T_{\beta}$.

If we apply the transform $k^{n}=h$ in (1.4), then we obtain the following problem,

$$
\left\{\begin{array}{l}
h_{t}=B(h)\left(h_{x x}+\left(1-h^{1 / n}\right)^{-\alpha}\right),(x, t) \in(0, L) \times(0, T), \\
h_{x}(0, t)=0, h_{x}(L, t)=\left(1-h^{1 / n}(L, t)\right)^{-\beta}, t \in(0, T), \\
h_{0}(x)=k_{0}^{n}(x), x \in[0, L],
\end{array}\right.
$$

where $B(h)=n h^{(n-1) / n}>0$ for $h>0$. Instead of (1.4), we use (2.3) in the proof of the next theorem for convenience.

Theorem 2.2. $x=L$ is the single quenching point if $h_{0}(x)\left(k_{0}(x)\right)$ satisfies (2.1) and (2.2).

Proof. Let $w \in(0, L), \rho \in(0, T)$ and $\xi>0$. Define

$$
\Theta(x, t)=h_{x}-\xi(x-(L-w)) \text { in }[L-w, L] \times[\rho, T) .
$$

$\Theta(x, t)$ supplies

$\Theta_{t}-B(h) \Theta_{x x}-\alpha\left(1-h^{1 / n}\right)^{-\alpha-1} \Theta=B^{\prime}(h) h_{x} \frac{h_{t}}{B(h)}+\xi \alpha\left(1-h^{1 / n}\right)^{-\alpha-1}(x-(L-w))>0$, in $(L-w, L) \times(\rho, T)$. Therefore, $\Theta(x, t)$ can't have a negative interior minimum using the maximum principle. Let $\xi$ be a sufficient small constant. We have $\Theta(x, \rho)>0$ since $h_{x}(x, t)>$ 0 in $(0, L] \times(0, T)$. Otherwise, we get

$$
\begin{aligned}
\Theta(L-w, t) & =h_{x}(L-w, t)>0, \\
\Theta(L, t) & =\left(1-h^{1 / n}(L, t)\right)^{-\beta}-\xi w>1-\xi w>0,
\end{aligned}
$$

where $\xi$ is a sufficient small constant and $t \in(\rho, T)$. From the maximum principle, we achieve that $\Theta(x, t)>0$, i.e. $h_{x}>\xi(x-(L-w)),(x, t) \in[L-w, L] \times[\rho, T)$. Taking integral with respect to $x$ from $(L-w)$ to $L$, we obtain that

$$
h(L-w, t)<h(L, t)-\frac{\xi w^{2}}{2}<1-\frac{\xi w^{2}}{2} .
$$

$h$ doesn't quench in $[0, L)$ in (2.3). So, $k$ doesn't quench in $[0, L)$ in (1.4). 
Theorem 2.3. $\lim _{t \rightarrow T_{\beta}^{-}} k_{t}(L, t) \rightarrow \infty$ if $k_{0}(x)$ satisfies (2.1) and (2.2).

Proof. Let $\chi$ be a positive constant. We will prove this theorem as in [1]. Assume that $k_{t}$ is bounded on $[0, L] \times[0, T)$. Thus,

$$
k_{t}<\chi \rightarrow\left(k^{n}\right)_{x x}+(1-k)^{-p}<\chi \Rightarrow\left(k^{n}\right)_{x x}<\chi
$$

Taking integral respect to $x$ from $x$ to $L$, and then from 0 to $L$, we get the following inequality

$$
L(1-k(L, t))^{-\beta}<\left(\chi L^{2}\right) / 2+k^{n}(L, t)-k^{n}(0, t) .
$$

This is a contradiction. Because, the right-hand side is finite while the left-hand side tends to infinity as $t \rightarrow T^{-}$. Therefore, $k_{t}$ blows up somewhere.

\subsection{Estimates for the quenching time}

In this subsection, we aim to get upper bound estimates for the quenching time by the help of lower solutions of (1.4).

Definition 1. A lower solution for (1.4) is called a function $\zeta \in C([0, L] \times[0, T)) \cap C^{2,1}((0, L) \times$ $(0, T))$ that satisfies the following inequalities:

$$
\left\{\begin{array}{l}
\zeta_{t}-\left(\zeta^{n}\right)_{x x} \leq(1-\zeta)^{-\alpha}, 0<x<L, 0<t<T, \\
\left(\zeta^{n}\right)_{x}(0, t)=0,\left(\zeta^{n}\right)_{x}(L, t) \leq(1-\zeta(L, t))^{-\beta}, 0<t<T, \\
\zeta(x, 0) \leq k_{0}(x), 0 \leq x \leq L .
\end{array}\right.
$$

It is an upper solution when the reverse inequalities are satisfied.

Lemma 2.1. Suppose a lower solution of (1.4) in $[0, L] \times[0, T)$ is $\zeta$. Then we get $k \geq \zeta$ in $[0, L] \times$ $[0, T)$.

The proof of Lemma 2.1 is a trivial modification of this in [6].

Theorem 2.4. If $1<n \leq 1 / L$ and $\alpha \geq \beta$, then $x=L(<1)$ is a quenching point.

Proof. Let's define

$$
\zeta(x, t)=1-\left(\frac{(\beta+1)\left(L^{2}-x^{2}+\rho-t\right)}{2}\right)^{1 /(\beta+1)} \text { in }[0, L] \times[0, \rho],
$$

where $\rho=2(1-m)^{\beta+1} /(\beta+1)$ and $m=\min _{x \in[0, L]} k_{0}(x) \geq 0$. We have

$$
\zeta_{t}-\left(\zeta^{n}\right)_{x x}-(1-\zeta)^{-\alpha}
$$




$$
\begin{aligned}
= & \frac{1}{2}\left(\frac{(\beta+1)\left(L^{2}-x^{2}+\rho-t\right)}{2}\right)^{-\frac{\beta}{\beta+1}}-\left(\frac{(\beta+1)\left(L^{2}-x^{2}+\rho-t\right)}{2}\right)^{-\frac{\alpha}{\beta+1}} \\
& -n\left[1-\left(\frac{(\beta+1)\left(L^{2}-x^{2}+\rho-t\right)}{2}\right)^{\frac{1}{\beta+1}}\right]^{n-1}\left(\frac{(\beta+1)\left(L^{2}-x^{2}+\rho-t\right)}{2}\right)^{-\frac{\beta}{\beta+1}} \\
& -n(n-1) x^{2}\left[1-\left(\frac{(\beta+1)\left(L^{2}-x^{2}+\rho-t\right)}{2}\right)^{\frac{1}{\beta+1}}\right]^{n-2}\left(\frac{(\beta+1)\left(L^{2}-x^{2}+\rho-t\right)}{2}\right)^{-\frac{2 \beta}{\beta+1}} \\
& -n \beta x^{2}\left[1-\left(\frac{(\beta+1)\left(L^{2}-x^{2}+\rho-t\right)}{2}\right)^{\frac{1}{\beta+1}}\right]^{n-1}\left(\frac{(\beta+1)\left(L^{2}-x^{2}+\rho-t\right)}{2}\right)^{-\frac{2 \beta+1}{\beta+1}} \\
\leq & 0
\end{aligned}
$$

for $\alpha \geq \beta, n>1$ and $x \in(0, L), t \in(0, \rho]$. Also, the following inequalities apply due to the character of this problem

$$
\zeta(x, t)=1-\left(\frac{(\beta+1)\left(L^{2}-x^{2}+\rho-t\right)}{2}\right)^{1 /(\beta+1)}<1
$$

and

$$
0<\left(\frac{(\beta+1)\left(L^{2}-x^{2}+\rho-t\right)}{2}\right)^{1 /(\beta+1)}<1
$$

Further,

$$
\begin{aligned}
\left(\zeta^{n}\right)_{x}(0, t) & =0 \\
\left(\zeta^{n}\right)_{x}(L, t) & \leq(1-\zeta(L, t))^{-\beta}
\end{aligned}
$$

for $n \leq 1 / L$ and $t \in(0, \rho]$. Furthermore,

$$
\zeta(x, 0)=1-\left(\frac{(\beta+1)\left(L^{2}-x^{2}+\rho-t\right)}{2}\right)^{1 /(\beta+1)} \leq 1-\left(\frac{(\beta+1) \rho}{2}\right)^{1 /(\beta+1)}=m
$$

for $x \in[0, L] . \zeta(x, t)$ is a lower solution from definition 1 . In addition, at $t=\rho$ and $x=L$, we get

$$
\zeta(L, \rho)=1
$$

Thus, we get

$$
k(L, \rho) \geq \zeta(L, \rho)=1
$$

by Lemma 2.1. Hence, $x=L$ is a quenching point. Also, we get $L<1$ since $n>1$ and $n \leq 1 / L$. 
Remark 3. We can easily calculate an upper bound for the quenching time. From Theorem 2.4, maximum upper bound is $T_{\beta}=2 /(\beta+1)$ (for $\alpha \geq \beta$ and $m=0$ ). From Remark $1, k_{0}(x)=$ $\frac{5}{4} x^{\frac{5}{8}}$ (for $\alpha \geq \beta=0.29827, L=1 / 2$ and $n=2$ ), then we have $T_{\beta}=1.5405$. Finally, we obtain a quenching rate

$$
k(L, T) \geq 1-C_{1}(T-t)^{1 /(\beta+1)} \text { in }[0, L] \times[0, T],
$$

where $C_{1}=\left(\frac{\beta+1}{2}\right)^{1 /(\beta+1)}$.

Theorem 2.5. An upper bound for the quenching time is $(1-m)^{\alpha+1} /(\alpha+1)$ if $k_{0}(x)$ satisfies (2.1) and (2.2).

Proof. We benefit from the following problem:

$$
\begin{gathered}
\zeta_{t}=\left(\zeta^{n}\right)_{x x}+(1-\zeta)^{-\alpha},(x, t) \in(0, L) \times(0, T), \\
\left(\zeta^{n}\right)_{x}(0, t)=0=\left(\zeta^{n}\right)_{x}(L, t), t \in(0, T), \\
\zeta(x, 0)=k_{0}(x), x \in[0, L] .
\end{gathered}
$$

By Definition 1, $\zeta$ is a lower solution for the problem (1.4). We suppose that $\zeta_{x}(t)=\zeta_{x x}(t)=$ 0 and $\zeta(0) \leq k_{0}(x)$ on $[0, L]$. Therefore, we focus the following initial-value problem instead of the above problem

$$
\begin{aligned}
& \zeta^{\prime}(t)=(1-\zeta(t))^{-\alpha} \text { for } t>0, \\
& \zeta(0)=m .
\end{aligned}
$$

So, taking integral respect $t$ from $t$ to $T$ in (2.5), we obtain that an upper bound for the quenching time is

$$
T \leq(1-m)^{\alpha+1} /(\alpha+1)
$$

The theorem is proved.

Remark 4. From Theorem 2.5, if we select $n=2, L=1 / 2, k_{0}(x)=\frac{5}{4} x^{\frac{5}{8}}$ as in Remark 1 , then we obtain that a maximum upper of quenching time $T_{\beta}$ is $1 /(\alpha+1)$ as in Remark 3 .

\section{Quenching for (1.5)}

In this section, the proof of the following lemma and theorems are congruent to that in Section 2 and Section 3 in [1]. 


\subsection{Quenching properties}

In this subsection, we examine to get single quenching point for (1.5).

Definition 2. $\zeta$ is called a lower solution of (1.5) if $\zeta \in C([0, L] \times[0, T)) \cap C^{2,1}((0, L) \times$ $(0, T))$ satisfies the following inequalities:

$$
\begin{aligned}
& \zeta_{t} \leq\left(\zeta^{n}\right)_{x x}, 0<x<L, 0<t<T, \\
& \left(\zeta^{n}\right)_{x}(0, t) \geq(1-\zeta(0, t))^{-\alpha},\left(\zeta^{n}\right)_{x}(L, t) \leq(1-\zeta(L, t))^{-\beta}, 0<t<T, \\
& \zeta(x, 0) \leq k_{0}(x), 0 \leq x \leq L .
\end{aligned}
$$

It is an upper solution when the reverse inequalities are satisfied.

Lemma 3.1. Assume $r=r\left(x, t, r_{0}\right)$ and $p=p\left(x, t, p_{0}\right)$ are solutions of (1.5) with $r_{0}=r_{0}(x)$ and $p_{0}=p_{0}(x)$, respectively. If $r_{0} \leq p_{0}<1$, then $r \leq p$ on $[0, L] \times[0, T)$.

The proof of Lemma 3.1 is a trivial modification of Theorem 1 given in [1].

If we apply the transform $k^{n}=h$ in (1.5), then we get following problem:

$$
\left\{\begin{array}{l}
h_{t}=B(h) h_{x x}, 0<x<L, 0<t<T, \\
h_{x}(0, t)=\left(1-h^{1 / n}(0, t)\right)^{-\alpha}, h_{x}(L, t)=\left(1-h^{1 / n}(L, t)\right)^{-\beta}, 0<t<T, \\
h_{0}(x)=k_{0}^{n}(x), 0 \leq x \leq L,
\end{array}\right.
$$

where $B(h)=n h^{(n-1) / n}>0$ for $h>0$. Instead of (1.5), we use (3.1) in the proofs of the next following remark and theorems for convenience.

Remark 5. It is easily proved that, if $B\left(h_{0}\right) h_{0}^{\prime \prime}(x) \geq 0$ in $[0, L]$, then $h_{t} \geq 0$ in $[0, L] \times(0, T)$. Also, $h_{t}>0$ in $(0, L) \times(0, T)$ using the strong maximum principle.

Theorem 3.1. If $B\left(h_{0}\right) h_{0}^{\prime \prime}(x) \geq 0$ in $[0, L]$, then (3.1) has only quenching point $x=L$.

Proof. We know that $h_{x}(0, t)=\left(1-h^{1 / n}(0, t)\right)^{-\alpha}>1$ and $h_{x x}=h_{t} /(B(h))>0$ in $(0, L) \times$ $(0, T)$. Thus, $h_{x}$ is an non-decreasing function and so, $h_{x}(x, t)>1 \mathrm{in}(0, L) \times(0, T)$. Assume $\xi \in(0, L)$. Taking integral this inequality with respect to $x$ from $L-\xi$ to $L$, we obtain

$$
h(L-\xi, t)<h(L, t)-\xi<1-\xi .
$$

So $h$ hasn't quench point in $[0, L)$. This completes the proof.

Theorem 3.2. Assume that $h(x, t)$ quenches finite time. Thus, $h_{t}$ blows up at the same time. 
Proof. Assume that $h_{t}$ is bounded holds. Namely $h_{t}<\chi$ on $[0, L] \times[0, T)$ since $\chi>0$. We get

$$
h_{x x}<\frac{\chi}{n} h^{(1-n) / n} .
$$

Multiplying the inequality by $h_{x}$, then integrating the inequality with respect to $x$ from 0 to $L$, we obtain

$$
\frac{1}{2}\left(\left(1-h^{1 / n}(L, t)\right)^{-\beta}-\left(1-h^{1 / n}(0, t)\right)^{-\alpha}\right)<\chi\left(h^{1 / n}(L, t)-h^{1 / n}(0, t)\right) .
$$

We see that the left-hand side tends to positive infinity, while the right-hand side is finite if $T_{\beta}$ is finite quenching time, as $t \rightarrow T_{\beta}^{-}$. This is a contradiction. Hence, we get $\lim _{t \rightarrow T_{\beta}^{-}} h_{t}(L, t)=\infty$ from Theorem 3.1 .

\subsection{Steady State}

In this subsection, we examine the steady state of (1.5)

$$
\left(K^{n}\right)_{x x}=0,\left(K^{n}\right)_{x}(0)=(1-K(0))^{-\alpha},\left(K^{n}\right)_{x}(L)=(1-K(L))^{-\beta} .
$$

In (3.2), we apply the transform $K^{n}=H$, then we get the following problem:

$$
H_{x x}=0, H_{x}(0)=\left(1-H^{1 / n}(0)\right)^{-\alpha}, H_{x}(L)=\left(1-H^{1 / n}(L)\right)^{-\beta} .
$$

We have

$$
H=(I+M x)^{n}
$$

where

$$
n M I^{n-1}=(1-I)^{-\alpha}, n M(I+M L)^{n-1}=(1-(I+M L))^{-\beta},
$$

which gives

$$
L(I)=(1-2 I) / M,
$$

since $\alpha=\beta=n-1$. We note that $L(I)>0$ for $0 \leq I<1 / 2$ implies $\alpha=\beta=n-1$. Also, we get maximum value of $L(I)=1 / M$ for $I=0$. We call $M=\max L(I)$.

Lemma 3.2. (3.2) has a solution $h(\leq 1)$ since $0 \leq I<1 / 2$ and $\alpha=\beta=n-1$.

Proof. If $\alpha=\beta=n-1$, then (3.3) has a solution if and only if $0<L \leq M$ since $0 \leq I<$ $1 / 2$.

Theorem 3.3. $h$ exists globally since $h_{0} \leq H(0), \alpha=\beta=n-1$ and $L \in(0, M]$.

Proof. By definition 2 and Lemma 3.1, $h \leq H$. Hence $h$ exists globally. So $k$ exists globally. 


\section{References}

[1] C. Y. Chan, S. I. Yuen, Parabolic problems with nonlinear absorptions and releases at the boundaries, Appl. Math. Comput., 121 (2001), 203-209.

[2] K. Deng and M. Xu, Quenching for a nonlinear diffusion equation with singular boundary condition, Z. Angew. Math. Phys., 50 (4) (1999), 574-584.

[3] Z. Jiang, S. Zheng and X. Song, Blow-up analysis for a nonlinear diffusion equation with nonlinear boundary conditions, Appl. Math. Letters, 17 (2004), 193-199.

[4] N. Ozalp and B. Selcuk, Blow up and quenching for a problem with nonlinear boundary conditions, Electron. J. Diff. Equ., 2015 (192) (2015), 1-11.

[5] N. Ozalp and B. Selcuk, The quenching behavior of a nonlinear parabolic equation with a singular boundary condition, Hacettepe Journal of Mathematics and Statistics, 44 (3) (2015), $615-621$.

[6] C. V. Pao, Singular reaction diffusion equations of porous medium type, Nonlinear Analysis, 71 (2009), 2033-2052.

[7] B. Selcuk and N. Ozalp, The quenching behavior of a semilinear heat equation with a singular boundary outflux, Quart. Appl. Math., 72 (4) (2014), 747-752.

[8] B. Selcuk and N. Ozalp, Quenching behavior of semilinear heat equations with singular boundary conditions, Electron. J. Diff. Equ., 2015 (311) (2015), 1-13.

[9] B. Selcuk and N. Ozalp, Quenching behavior of nonlinear diffusion equation with singular boundary outfluxes, Turkish Journal of Mathematics and Computer Science, 8 (2018), $65-$ 69.

[10] J. L. Vazquez, The porous medium equation: Mathematical Theory, Oxford Science Publications, (2007).

[11] Z. Zhang and Y. Li, Quenching rate for the porous medium equation with a singular boundary condition, Applied Mathematics, 2 (2011), 1134-1139.

[12] Y. Zhi and C. Mu, The quenching behavior of a nonlinear parabolic equation with a nonlinear boundary outflux, Appl. Math. Comput, 184 (2007), 624-630.

[13] L. Zhu, The quenching behavior of a quasilinear parabolic equation with double singular sources, C. R. Acad. Sci. Paris, Ser. I, 356 (7) (2018), 725-731. 
Burhan Selcuk Department of Computer Engineering, Karabuk University, Karabuk, Turkey E-mail: bselcuk@karabuk.edu.tr 